Check for updates

Cite this: RSC Adv., 2018, 8, 29022

\title{
Biofabricated BiOl with enhanced photocatalytic activity under visible light irradiation $\uparrow$
}

\author{
Seema Garg, ${ }^{* a}$ Mohit Yadav, (DD ab Amrish Chandra, ${ }^{c}$ Sameer Sapra, (D) d \\ Soniya Gahlawat, ${ }^{d}$ Pravin P. Ingole, (D) ${ }^{d}$ Zsolt Pap $^{\text {ef }}$ and Klara Hernadi ${ }^{9}$
}

In the recent past, there has been a large-scale utilization of plant extracts for the synthesis of various photocatalysts. The biofabrication technology eliminates the usage of harmful chemicals and serves as an eco-friendly approach for environmental remediation. Herein, a comparative analysis between bismuth oxyiodide synthesized via Azadirachta indica (neem) leaf extract (BiOl-G) and without leaf extract ( $\mathrm{BiOl}-\mathrm{C}$ ) has been envisaged. The $\mathrm{BiOl}-\mathrm{G}$ and $\mathrm{BiOl}-\mathrm{C}$ samples were characterized by spectral and microscopic techniques, which revealed that the Azadirachta indica assisted BiOl-G attained enhanced features over BiOl-C such as narrower band gap, large surface area, porosity, increased absorption range of visible light and effectual splitting of the photogenerated $\mathrm{e}^{-}-\mathrm{h}^{+}$pairs. Benefiting from these enhanced features, BiOl-G degraded methyl orange (MO), rhodamine $B(R h B)$, and benzotriazole (BT) at a significantly higher rate in comparison to $\mathrm{BiOl}-\mathrm{C}$. The degradation rate of $\mathrm{MO}, \mathrm{RhB}$ and $\mathrm{BT}$ by $\mathrm{BiOl}-\mathrm{G}$ was observed to be 1.3, 1.25 and 1.29 times higher in comparison to BiOl-C. Moreover, BiOl-G displayed high stability upto five cycles of the photocatalytic activity, which endow its effectiveness as a highlyefficient green photocatalyst.

Received 2nd July 2018

Accepted 9th August 2018

DOI: $10.1039 / \mathrm{c} 8 \mathrm{ra0} 6661 \mathrm{~g}$

rsc.li/rsc-advances benghalensis (banyan tree) ${ }^{6}$ etc. has been broadly explored for the biofabrication of various nanostructures. Likewise, Azadirachta indica (neem) $)^{7-9}$ is widely known to possess reducing and stabilizing properties, as it comprises biologically active compounds i.e. phytochemicals. The broad amount of phytochemicals such as flavones, terpenoids, nimbin, quercetin, aldehydes, organic acids, ketones, and amides possess antioxidant and stabilizing properties, which are accountable for the regulation of size and providing stability to the nanostructures. ${ }^{10-12}$

In recent times, BiOI has intrigued researchers immensely owing to its unusual character and ability to perform under visible light. It has layered tetragonal matalockite geometry, characterized by $\left[\mathrm{Bi}_{2} \mathrm{O}_{2}\right]^{2+}$ slabs interleaved by double slabs of iodine atoms, determined its narrow band gap $(1.8 \mathrm{eV}) \cdot{ }^{13-16}$ To advance its photocatalytic performance, BiOI has been functionalized with various material like $\mathrm{TiO}_{2},{ }^{17,18} \mathrm{BiVO}_{4},{ }^{19} \mathrm{BiPO}_{4},{ }^{20}$ $\mathrm{WO}_{3},{ }^{21,22} \mathrm{Bi}_{2} \mathrm{~S}_{3},{ }^{23}$ carbon quantum dots, ${ }^{24} \mathrm{Au},{ }^{25} \mathrm{Ag}^{26}$ and $\mathrm{CdS}^{27}$ etc. However, these reports are mainly concentrated on the modulation and tuning of the optical and structural properties of BiOI, and some of these methods are not ecofriendly as their synthesis route involves the application of harmful solvents and stabilizers such as ethylene glycol, ${ }^{28}$ ethanol ${ }^{29}$ and $\mathrm{CTAB}^{30}$ etc. The consumption of such chemical solvents can even result in residual toxicity or may inflict secondary pollution.

Therefore, for the first time in our approach, BiOI has been synthesized by simple green route without any harmful chemical solvents. Alternatively, Azadirachta indica leaf extract was 
utilized to provide stabilization and controlling the size of the material. The as-prepared BiOI-G with the addition of leaf extract attained advantageous features such as higher surface area, porosity, adequate active interaction sites and efficacious partition of photogenerated charge carriers. All the features boosted the photocatalytic activity of BiOI-G towards RhB, MO and BT in comparison to BiOI-C. Moreover, this research methodology opens a pioneering design to progress our understanding of how plant extracts can be practically applied in the field of photocatalysis.

\section{Experimental}

\section{Materials}

All the chemicals and reagents such as bismuth nitrate pentahydrate $\left[\mathrm{Bi}\left(\mathrm{NO}_{3}\right)_{3} \cdot 5 \mathrm{H}_{2} \mathrm{O}\right]$, potassium iodide (KI), methyl orange, rhodamine $\mathrm{B}$ and benzotriazole were of analytical grade and used without any further purification.

\section{Synthesis of plant extract}

The Azadirachta indica (neem leaves) were collected locally within the Amity University campus, Noida, India. The leaves were washed with double distilled water to wipe out debris and other impurities. Then, the leaves were allowed to dry at room temperature and the plant extract was prepared using distilled water and utilized for experimental purpose.

\section{Synthesis of BiOI-G and BiOI-C}

BiOI-G was biofabricated by minor modifications in the hydrolysis route i.e. inclusion of aqueous neem leaf extract. Typically, $100 \mathrm{~mL}$ of $0.05 \mathrm{M} \mathrm{Bi}\left(\mathrm{NO}_{3}\right)_{3} \cdot 5 \mathrm{H}_{2} \mathrm{O}$ aqueous solution was added dropwise to $8 \mathrm{~mL}$ plant leaf extract with constant stirring. Then, the obtained solution was added dropwise to a $0.05 \mathrm{M}$ KI solution under continuous stirring for 1 hour at room temperature, and it was kept in an oven for 1 hour at $60{ }^{\circ} \mathrm{C}$. The resulting product was separated via centrifugation followed by washing with ethanol and double distilled water thrice to eliminate the impurities, and finally, it was dried in an oven at $60{ }^{\circ} \mathrm{C}$. BiOI-C was synthesized with same methodology; however, the usage of plant extract was avoided.

\section{Characterizations}

The X-ray diffraction patterns were measured on a Bruker D2Phaser Diffractometer with a $\mathrm{Cu} \mathrm{K} \alpha$ radiation source $(\lambda=$ $1.5418 \AA$ ). The particle size, morphology and elemental analysis of BiOI-G and BiOI-C were inspected by Zeiss-Sigma VP field emission scanning electron microscope, FEI Technai G2 X-Twin TEM $(200 \mathrm{kV})$ and EDS (Bruker). Specific surface areas were determined by BET method for which nitrogen adsorptiondesorption studies were analyzed at $77 \mathrm{~K}$ using a Quanta Chrome NOVA 1000. The functional groups were recognized by FTIR (Agilent Cary 630). The optical properties were evaluated by both UV-Vis diffuse reflectance spectra (Shimadzu UV-1800) and photoluminescence spectra (Shimadzu RF-5301).

\section{Photocatalytic activity measurements}

The photocatalytic activity of the as-prepared BiOI-C and BiOI-G samples was evaluated for the disintegration of MO, RhB and BT. Experimental details were as follows: four compact fluorescent lamps $28 \mathrm{~W}$ each were utilized as visible light source for the photocatalyst and $50 \mathrm{~g} \mathrm{~L}^{-1} \mathrm{NaNO}_{2}$ solution was utilized as a UV cutoff filter. Initially, $50 \mathrm{mg}$ of BiOI-G and BiOI-C were added to $100 \mathrm{~mL}$ of $20 \mathrm{mg} \mathrm{L}^{-1}$ MO solution (or $20 \mathrm{mg} \mathrm{L}^{-1} \mathrm{RhB}$ or $10 \mathrm{mg} \mathrm{L}^{-1} \mathrm{BT}$ ) in a $250 \mathrm{~mL}$ beaker separately and the $\mathrm{pH}$ of the initial solution was about 7. Primarily, the suspension was stirred on a magnetic stirrer in dark for 60 minutes to ensure appropriate adsorption-desorption equilibrium. Then, the suspension was subjected to visible light irradiation under constant stirring. After certain time intervals, $2 \mathrm{~mL}$ of the suspension was withdrawn and the photocatalyst particles were separated through centrifugation at $10000 \mathrm{rpm}$. The absorbance of the supernatant was measured by UV-Vis spectrophotometer at $465 \mathrm{~nm}$ for $\mathrm{MO}$ and at $550 \mathrm{~nm}$ for RhB respectively. The photocatalytic concentration was varied from 50-125 mg/ $100 \mathrm{~mL}$, respectively. The photogenerated intermediates during the disintegration of BT were separated and measured by High performance liquid chromatography (HPLC, Shimadzu). The enable C18G $(250 \times 4.6 \mathrm{~mm}$ i.d., $5 \mu \mathrm{m})$ reversed phase column was utilized and a mixture of water and ethanol $(40: 60 \mathrm{v} / \mathrm{v})$ was used as a mobile phase and the flow rate was

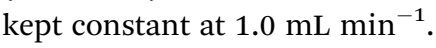

\section{Radical trapping study}

At the time of photocatalytic reaction, holes $\left(\mathrm{h}^{+}\right)$, hydroxy radicals $\left({ }^{\circ} \mathrm{OH}\right)$ and superoxide radicals $\left({ }^{\circ} \mathrm{O}_{2}{ }^{-}\right)$were produced and were evaluated by adding $1 \mathrm{mM}$ sodium oxalate (a scavenger of $\mathrm{h}^{+}$), $1 \mathrm{mM}$ isopropanol (a scavenger of ${ }^{\circ} \mathrm{OH}$ ) and $1 \mathrm{mM}$ benzoquinone (a scavenger of ${ }^{\circ} \mathrm{O}_{2}{ }^{-}$). The same methodology mentioned above was implemented in this activity.

\section{Electrochemical studies}

The electrochemical experiments were implemented on a $\mathrm{CHI}$ $608 \mathrm{E}$ electrochemical system using a standard three electrode system i.e. a working electrode, a saturated $\mathrm{Hg} / \mathrm{HgO}$ reference electrode and a counter electrode (platinum wire). The working electrode was prepared by drop-casting method: briefly, $2 \mathrm{mg}$ of material was ultrasonically dispersed in $100 \mu \mathrm{L}$ ethanol and 10 $\mu \mathrm{L}$ NAFION, of which $10 \mu \mathrm{L}$ was then drop-casted on a piece of ITO slice with a fixed area of $1 \mathrm{~cm}^{2}$. The properties were evaluated with a $150 \mathrm{~W}$ Xe arc lamp and $0.1 \mathrm{M} \mathrm{KOH} \mathrm{(pH} \mathrm{12.8)} \mathrm{as} \mathrm{an}$ electrolyte. The electrochemical impedance spectroscopy (EIS) was recorded at $0 \mathrm{~V}$ versus $\mathrm{Hg} / \mathrm{HgO}$ from $1 \mathrm{MHz}$ to $10 \mathrm{MHz}$, respectively.

\section{Results and discussion}

\section{Characterization of BiOI-C \& BiOI-G photocatalysts}

$\mathrm{XRD}$ was employed to identify the phase composition and crystal structure of BiOI-C and BiOI-G samples. As shown in Fig. 1(a), the diffraction patterns of both the samples are quite similar and all the identified peaks corresponding to (002), 
(102), (110), (112), (004), (200), (114) and (212) planes are in agreement with JCPDS card no. 73-2062 (ref. 31) and can be indexed to the tetragonal phase of BiOI. Interestingly, with the incorporation of Azadirachta indica leaf extract in the host matrix of BiOI, the overall intensity of the XRD peaks decreased, suggesting the degradation of crystallinity for BiOI-G sample. The possible reason for the decrease in crystallinity could be the generation of crystals defects in BiOI-G lattice, which resulted in the charge imbalance and changed stoichiometry of the sample. ${ }^{9,32}$ Furthermore, in the XRD spectrum of BiOI-G full width at half maximum (FWHM) seems to be widened, indicating a subsequent decrease in the crystallite size of BiOI-G in comparison to BiOI-C. The average crystallite sizes of BiOI-G and BiOI-C were calculated using Debye Scherer's equation (ESI, Section $\mathrm{S} 1 \dagger$ ) and they were determined to be $4.7 \mathrm{~nm}$ and $12.5 \mathrm{~nm}$, respectively. Moreover, no extra peaks appeared in the XRD pattern of BiOI-G, suggesting that the Azadirachta indica leaf extract was uniformly incorporated in the BiOI-G matrix.
The XPS spectra of BiOI-G and BiOI-C were carried out to inspect the chemical composition and surface chemical states. As depicted in Fig. 1(c), both BiOI-G and BiOI-C were comprised of $\mathrm{Bi}, \mathrm{O}$, and I elements which is consistent with their chemical composition. Fig. 1(d) displays the high-resolution Bi 4 f spectra of BiOI-G and BiOI-C, the peaks at $158.62 \mathrm{eV}$ and $163.74 \mathrm{eV}$ were attributed to $\mathrm{Bi} 4 \mathrm{f}_{7 / 2}$ and $\mathrm{Bi} 4 \mathrm{f}_{5 / 2}$, which are characteristic of the $\mathrm{Bi}^{3+}$ in the BiOI samples. ${ }^{33}$ For BiOI-C, the $\mathrm{O} 1 \mathrm{~s}$ profile was resolved into two identical peaks positioned at $529.6 \mathrm{eV}$ and $530.4 \mathrm{eV}$, indicating the presence of two individual $\mathrm{O}$ species in both the samples. The $\mathrm{O} 1 \mathrm{~s}$ peak originated at $529.4 \mathrm{eV}$ was ascribed to crystal lattice $\mathrm{O}$ atoms $(\mathrm{Bi}-\mathrm{O})$; while the peak occurred at $530.4 \mathrm{eV}$ was credited to the $\mathrm{Bi}-\mathrm{O}$ bonds in the $\left[\mathrm{Bi}_{2} \mathrm{O}_{2}\right]$ slabs of BiOI. For the BiOI-G the O 1 s peak at $530.7 \mathrm{eV}$ shifted slightly, which could possibly be as a result of minor change in the $\mathrm{O}$ environment due to the usage of plant extract. ${ }^{34}$ In the XPS spectrum of I 3d [Fig. 1(f)], the two distinguished peaks at $618.09 \mathrm{eV}$ and $630.12 \mathrm{eV}$ accounts for the binding
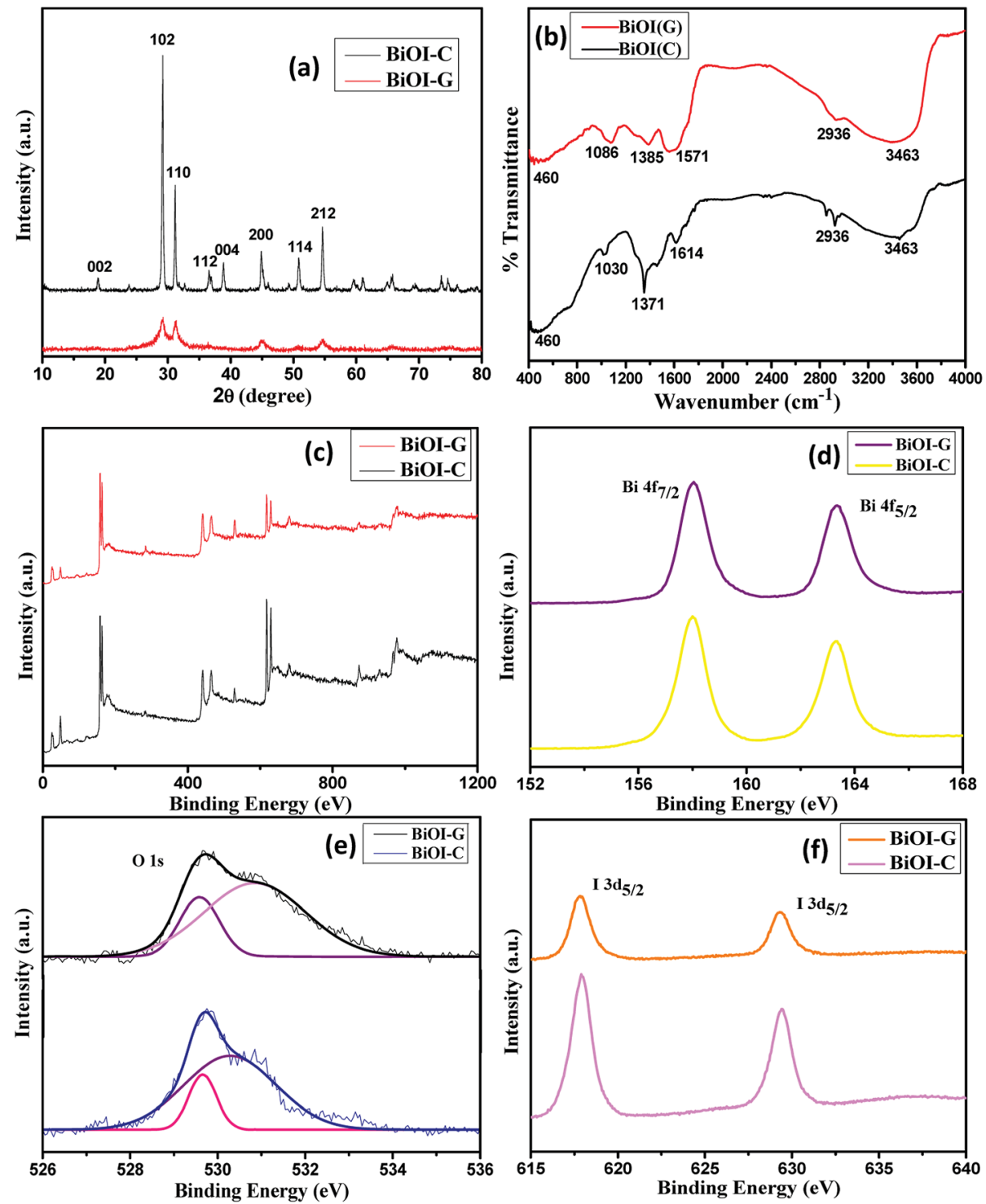

Fig. 1 (a) XRD patterns, (b) FTIR spectra, XPS spectra (c) survey of the samples, (d) Bi 4f, (e) O 1s and I 3d, (f) BiOl-C and BiOl-G. 

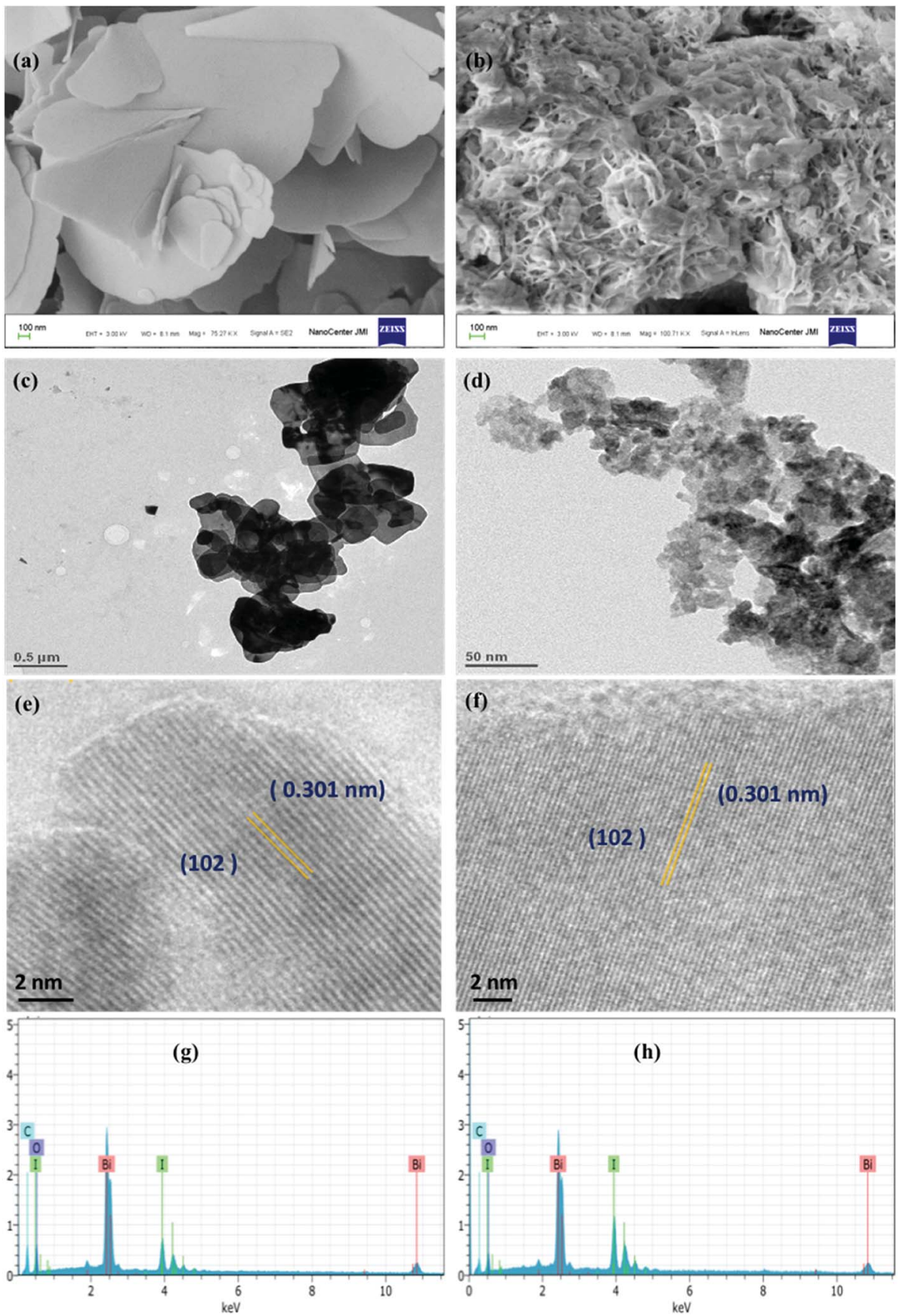

Fig. 2 ( $a$ and b) FESEM images, (c and d) TEM images, (e and f) HR-TEM images, ( $g$ and h) EDS images of BiOl-C (left) and BiOl-G (right).

energies of $\mathrm{I}_{3 / 2}$ and $\mathrm{I} 3 \mathrm{~d}_{3 / 2}$, confirming the presence of $\mathrm{I}^{-}$in BiOI-C and BiOI-G. ${ }^{35}$ Hence, the results established the existence of $\mathrm{Bi}$, I and $\mathrm{O}$ elements with their most likely oxidation states in the BiOI-G and BiOI-C structures. The results are consistent with the XRD analysis.

To inspect the surface functional groups existing in the asprepared BiOI-C and BiOI-G, the FTIR spectra were recorded in the range of $400-4000 \mathrm{~cm}^{-1}$. As presented in Fig. 1(b) the peaks originated at $3463 \mathrm{~cm}^{-1}$ and $460 \mathrm{~cm}^{-1}$ are credited to $\mathrm{O}-\mathrm{H}$ stretching vibration and $\mathrm{Bi}-\mathrm{O}$ chemical stretching vibration mode in the crystal tetragonal phase of BiOI respectively. ${ }^{12,36}$
The morphology of BiOI-C and BiOI-G nanostructures was investigated by field emission scanning electron microscopy. As depicted in Fig. 2(a and b), as-prepared samples possessed nanoplate like structure and the size of the BiOI-C nanoplates seem to be significantly bigger in comparison to those of BiOIG. Furthermore, the incorporation of Azadirachta indica leaf extract in the BiOI-G matrix has resulted in a web-like structure of the nanoplates and simultaneously high porosity can be observed. It suggests that BiOI-G is more likely to attain larger surface area as compared to BiOI-C, which could facilitate the contact area between the reactants and the BiOI-G surface. 

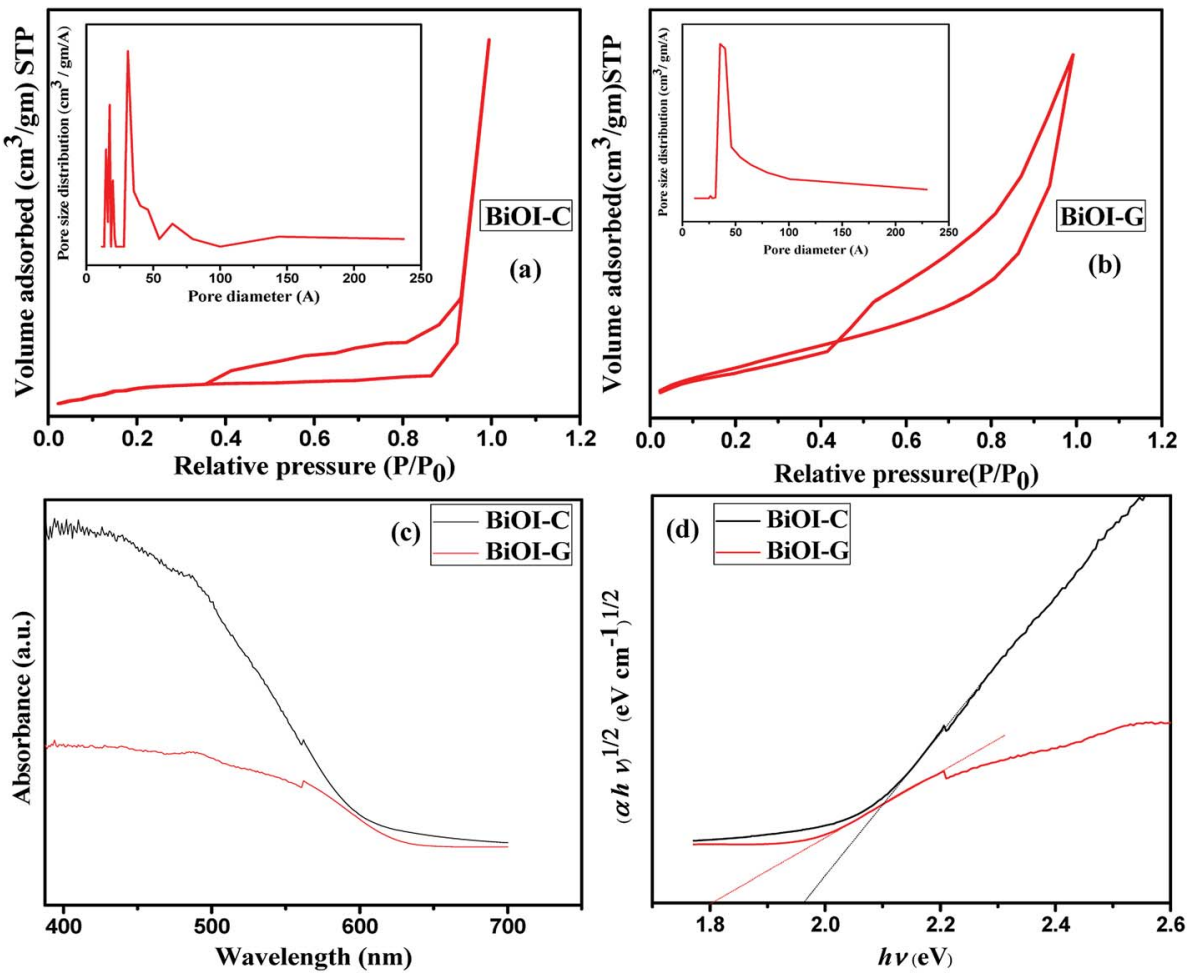

Fig. 3 (a and b) $\mathrm{N}_{2}$ adsorption-desorption isotherms and pore size distributions, (c and d) UV-Vis DRS and plot of $(\alpha h \nu)^{n} v s$. $h \nu$ of BiOI-C and BiOl-G.

To explore a better analysis of the BiOI-C and BiOI-G samples, TEM study was carried out as presented in Fig. 2(c and d), respectively. The TEM images further confirm the smaller size of BiOI-G than BiOI-C. To obtain a deeper view, the BiOI-C and BiOI-G samples were further characterized with HRTEM as presented in Fig. 2(e and f). Clearly, a highly crystalline pattern can be observed with clear lattice fringes in both the samples. The continuous lattice fringes with an interplanar lattice spacing of $0.301 \mathrm{~nm}$ matched well with the (102) atomic planes of the samples, respectively.

In addition, the chemical composition of the BiOI-C and BiOI-G samples was analyzed by energy dispersive X-ray spectroscopy as displayed in Fig. 2 ( $g$ and $h$ ), respectively. The results indicate that both BiOI-C and BiOI-G nano-spheres contain Bi, I and $\mathrm{O}$ elements, respectively.

The surface area and pore size distribution of the asprepared BiOI-G and BiOI-C samples were determined by nitrogen adsorption-desorption study as shown in Fig. 3(a and b), respectively. It can been seen, that both the photocatalysts exhibited the type IV isotherm with a distinct H3 hysteresis loop at high relative pressure indicating the mesoporous structure according to the IUPAC classification. ${ }^{37,38}$ The BET surface area and pore volume for BiOI-C were $2.507 \mathrm{~m}^{2} \mathrm{~g}^{-1}$ and $0.005 \mathrm{~m}^{3} \mathrm{~g}^{-1}$, and $18.070 \mathrm{~m}^{2} \mathrm{~g}^{-1}$ and $0.048 \mathrm{~m}^{3} \mathrm{~g}^{-1}$ for BiOI-G, respectively. The average Barrett-Joyner-Halenda (BJH) pore diameter of the BiOI-G and BiOI-C calculated from desorption isotherm study came out to be $3.111 \mathrm{~nm}$ and $3.551 \mathrm{~nm}$, respectively. Palatably, Azadirachta indica leaf extract sufficiently enriches the specific surface area of BiOI-G, which is upto nine times to that of the
BiOI-C. Moreover, the materials with large surface area tend to possess adequate active sites, which would absorb more active species and allows sufficient contact between the photocatalyst and the pollutants. The increased the contact area can boost the transport of reactant species and subsequently, improve the photocatalytic activity, which is also consistent with FESEM analysis. ${ }^{39}$

The optical absorption property of BiOI-G and BiOI-C were evaluated via UV-vis diffuse reflectance spectroscopy (DRS). As presented in Fig. 3(c), BiOI-C exhibited an absorption edge onset at $630 \mathrm{~nm}$, corresponding to a band gap of $1.97 \mathrm{eV}$ as computed by the Kubelka-Munk concept [Fig. 3(d)]. BiOI-G displayed a red-shift towards a deeper visible region exhibiting an absorption onset at around $688 \mathrm{~nm}$ and the band gap of BiOI-G was measured to be $1.8 \mathrm{eV}$. Consequently, BiOI-G under the irradiation of visible light would generate higher number of $\mathrm{e}^{-}-\mathrm{h}^{+}$pairs, ultimately resulting in superior photocatalytic activity. ${ }^{40}$

\section{Photocatalytic performance}

To inspect the photocatalytic performance of the BiOI-C and BiOI-G samples, MO and RhB with initial concentrations of $20 \mathrm{mg} \mathrm{L}^{-1}$ were taken as the dye pollutants. As presented in Fig. 4(a and g), the blank test confirmed that the self-photolysis of $\mathrm{MO}$ and $\mathrm{RhB}$ was negligible, suggesting that the dyes are chemically stable under visible light irradiation. In the dark experiment, about $8.91 \%$ and $12.11 \%$ of $\mathrm{MO}$, and $7.11 \%$ and $15.23 \%$ of RhB was adsorbed on the BiOI-C and BiOI-G surface. 

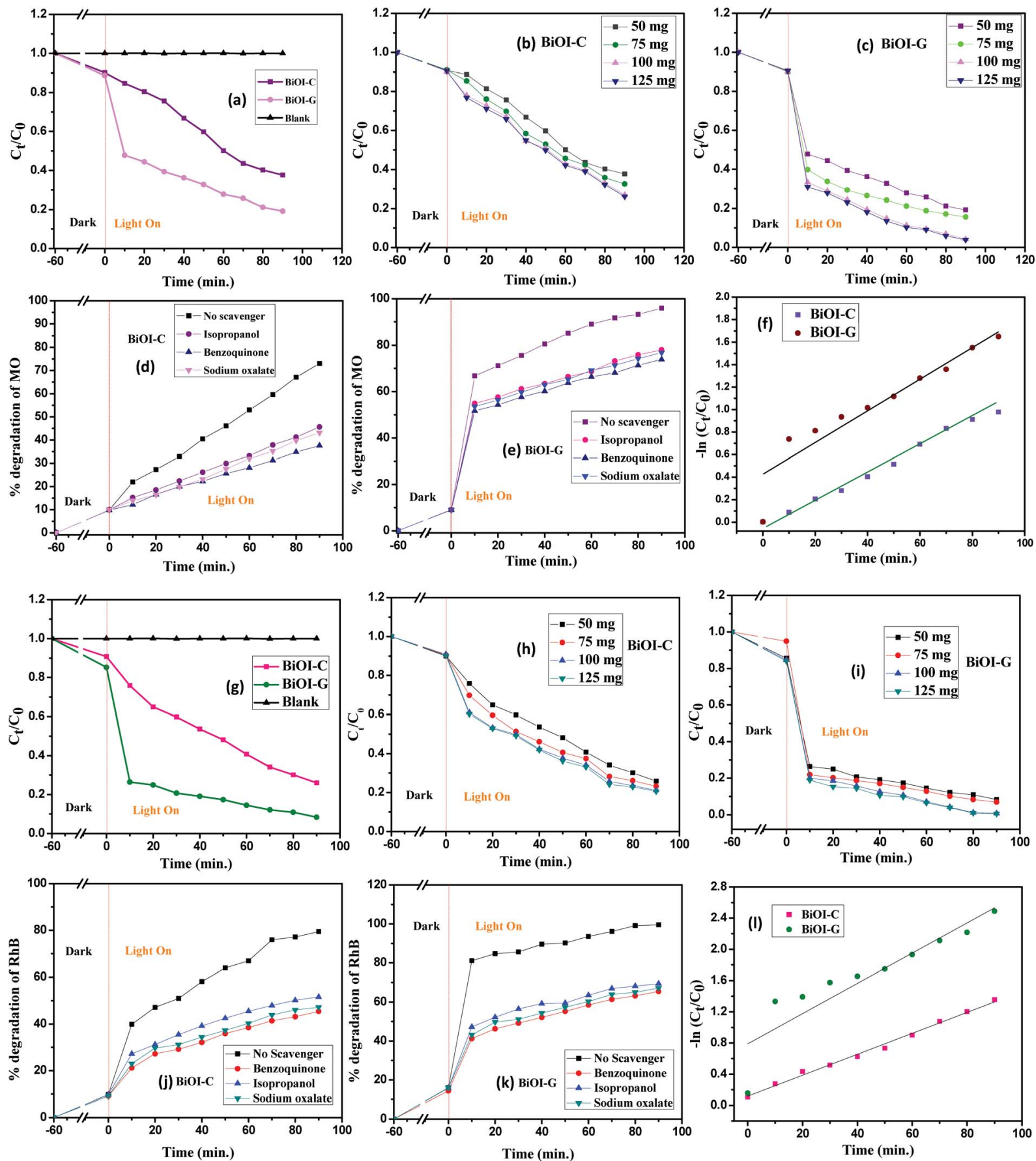

Fig. 4 Photodegradation efficacy of $\mathrm{MO}$ and $\mathrm{RhB}$ by BiOl-C and BiOl-G (a and g) with time, (b and $\mathrm{h}$ ) with different $\mathrm{BiOI}-\mathrm{C}$ concentrations, (c and i) with different $\mathrm{BiOl}-\mathrm{G}$ concentrations, ( $d$ and $\mathrm{j}$ ) impact of scavengers on $\mathrm{MO}$ and $\mathrm{RhB}$ degradation in presence of $\mathrm{BiOl-C}$ (e and $\mathrm{k}$ ), impact of scavengers on $\mathrm{MO}$ and $\mathrm{RhB}$ degradation in presence of BiOl-G, (f and l) Kinetic linear simulation curves of RhB and MO over the samples.

However, under visible light irradiation, 63.19\% and $81.56 \%$ MO degradation, while $74.09 \%$ and $91.77 \%$ RhB degradation was found by BiOI-C and BiOI-G within 90 minutes. To check the maximum degradation of the dyes was checked and the photocatalytic concentration was varied from 50-125 mg/100 mL. As presented in Fig. 4(b, c, h and i), with increase in photocatalytic concentration the degradation of $\mathrm{MO}$ and $\mathrm{RhB}$ further increased upto $73.89 \%$ and $95.15 \%$, and $79.93 \%$ and
99.47\% by BiOI-C and BiOI-G, respectively. Clearly, the photocatalytic efficacy of BiOI-G for MO was nearly $21 \%$ more in comparison to BiOI-C, while in case of RhB it was around $20 \%$, respectively.

A batch of experiments was then conducted to inspect the active species responsible for $\mathrm{MO}$ and $\mathrm{RhB}$ disintegration under visible light by the addition of three different scavengers (sodium oxalate for $\mathrm{h}^{+}$, isopropanol for ${ }^{\circ} \mathrm{OH}$ and benzoquinone 

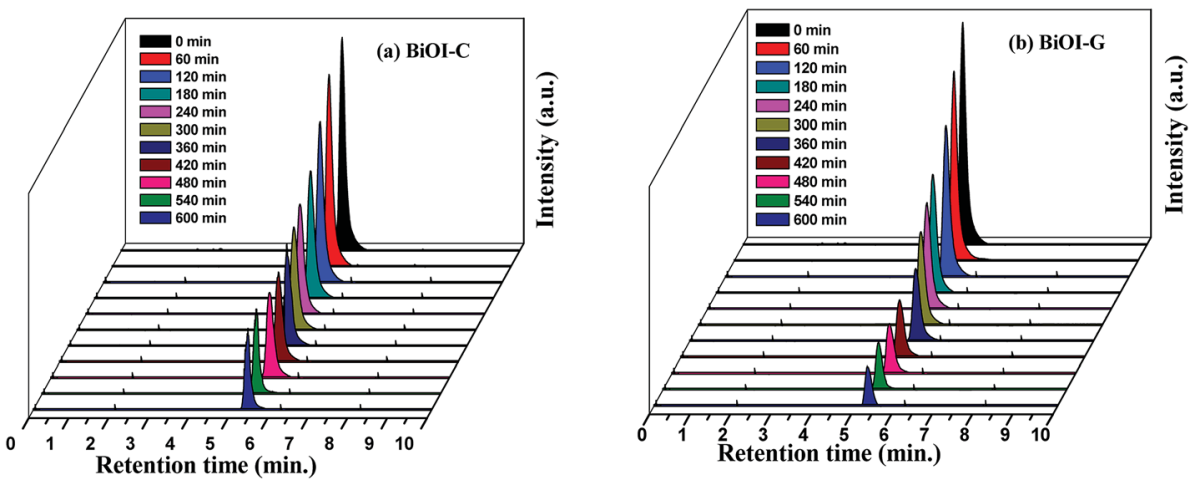

Fig. 5 HPLC spectra of benzotriazole degradation by (a) BiOl-C and (b) BiOl-G.

for ${ }^{\circ} \mathrm{O}_{2}{ }^{-}$). As presented in Fig. $4(\mathrm{~d}, \mathrm{e}, \mathrm{j}$ and $\mathrm{k}$ ), an obvious inhibition of the photocatalytic performance was observed, the disintegration rate was evidently decreased in all the cases. It suggests that all the three scavengers are playing a major part in the photo-oxidation process.

The photodisintegration activity was fit with a pseudo-first order kinetics model as presented in Fig. 4(f and l).

$$
-\ln \left(C_{t} / C_{0}\right)=k t
$$

where $C_{t}$ is the dye concentration at time $t, C_{0}$ is the initial dye concentration and $k$ is the rate constant. The rate constants for BiOI-C and BiOI-G in case of MO were obtained to be $0.013 \mathrm{~min}^{-1}$ and $0.028 \mathrm{~min}^{-1}$ and, $0.015 \mathrm{~min}^{-1}$ and $0.039 \mathrm{~min}^{-1}$ in case of $\mathrm{RhB}$, respectively. The higher rate constant means lower activation energy $\left(E_{\mathrm{a}}\right)$ and higher degradation rate; hence, BiOI-G has shown much higher photodegradation efficiency towards MO and RhB than BiOI-C.
To inspect the stability of BiOI-C and BiOI-G, the assynthesized samples after every 90 minutes of photocatalytic experiment with $\mathrm{MO}$ and $\mathrm{RhB}$ were collected via centrifugation followed by washing with double distilled water several times. Finally, the samples were reused in the photocatalytic reactions five times under the same optimized conditions (ESI, Section S2†).

In prospect of the dye sensitization, a typical colourless pollutant i.e. benzotriazole was also chosen to further inspect the photocatalytic performance of BiOI-C and BiOI-G (ESI, Section S3†). To explore the degraded products of the benzotriazole, the reaction intermediates were recognized during the photocatalytic process using HPLC. Fig. 5(a and b), display the disintegration proces of benzotriazole, where the decreasing peak of benzotriazole occurred at $5.7 \mathrm{~min}$. It can further be noticed that apart from the prominent peak of BT, no other peak was detected in both the cases, which suggests that
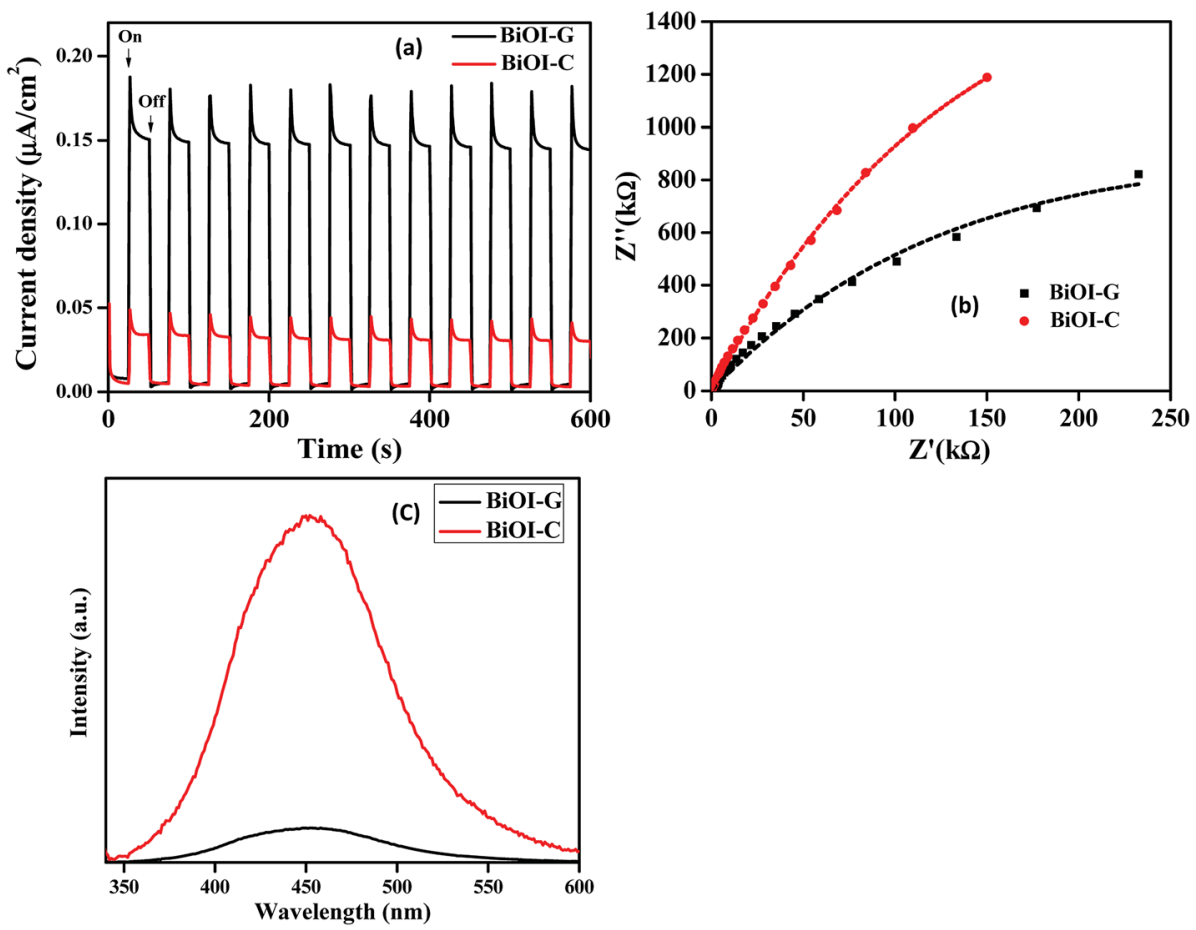

Fig. 6 (a) Transient photocurrent response, (b) EIS, and (c) PL spectra of BiOl-C and BiOl-G. 
benzotriazole is not dissociating into any harmful intermediate, it is rather getting suppressed with every time interval.

\section{Photocatalytic mechanism}

The valence band (VB) and conduction band (CB) of BiOI-G and BiOI-C were calculated using eqn $(2)^{41}$ and $(3)^{42}$

$$
\begin{gathered}
E_{\mathrm{VB}}=X-E^{\mathrm{e}}+0.5 E_{\mathrm{g}} \\
E_{\mathrm{CB}}=E_{\mathrm{VB}}-E_{\mathrm{g}}
\end{gathered}
$$

where, $X=$ absolute electronegativity of the semiconductor, $E^{\mathrm{e}}$ $=$ energy of free electrons on the hydrogen scale $(c a .4 .5 \mathrm{eV}), E_{\mathrm{g}}$ $=$ band gap of the semiconductor.

The $E_{\mathrm{VB}}$ and $E_{\mathrm{CB}}$ values for BiOI-G and BiOI-C were estimated to be $2.39 \mathrm{eV}$ and $0.59 \mathrm{eV}$, and $2.475 \mathrm{eV}$ and $0.5 \mathrm{eV}$, respectively.

To inspect the charge transfer, recombination and migration process in BiOI-C and BiOI-G, the photocurrent response and EIS studies were carried out. As presented in Fig. 6(a), BiOI-G showed considerably greater photocurrent intensity than BiOI-C. It can be interpreted that the BiOI-G favorably allows more effective parting of photogenerated $\mathrm{e}^{-}-\mathrm{h}^{+}$pair in comparison to BiOI-C. To assess more insights about the charge mobility in the BiOI$\mathrm{G}$ and BiOI-C electrodes, EIS analysis was carried out. As presented in Fig. 6(b), the Nyquist circle of BiOI-G is much smaller in diameter in comparison to BiOI-C, indicating a lower resistance and subsequently, much faster carrier mobility from the BiOI-G to the electrode in comparison to BiOI-C. ${ }^{43}$ As discussed above, the energy band structure of BiOI-G and BiOI-C was accounted for the different separation efficacy of the photogenerated $\mathrm{e}^{-}-\mathrm{h}^{+}$ pair. Therefore, BiOI-G displayed a slight downshift in the $\mathrm{CB}$, which resulted in production of photogenerated electrons to react with $\mathrm{O}_{2}$ to form ${ }^{\circ} \mathrm{O}_{2}{ }^{-}$. In contrast, the upshifting in the VB subsequently inhibited the $\mathrm{e}^{-}-\mathrm{h}^{+}$pair recombination by having an intrinsic control over the mobility of the holes and much faster transmission of electrons to the reactants. ${ }^{\mathbf{4 4 , 4 5}}$

To further justify the above result, photoluminescence (PL) spectra of BiOI-C and BiOI-G are presented in Fig. 6(c), respectively. Clearly, BiOI-G exhibited significantly lower PL intensity compared to BiOI-C, which implies that the photogenerated $\mathrm{e}^{-}-\mathrm{h}^{+}$pair in BiOI-G has been efficiently separated in comparison to BiOI-C. ${ }^{46}$

To identify the reasons for the much improved photocatalytic performance of BiOI-G, certain factors were addressed such as morphology, energy band diagram, transport of electrons and surface area. Firstly, the photocatalytic capability of a certain photocatalyst is directly associated to its morphology. From the earlier studies, BiOI-G was observed to be highly porous and has significantly higher surface area in comparison to BiOI-C.

Thus, the impact of morphology could be a primary reason for the difference noticed in the photocatalytic ability of the two samples. Now, considering the large surface area and adsorption capacity of BiOI-G, adequate number of photocatalytic reaction sites could have been another factor for the improved performance. Furthermore, the energy band structure of a material is associated to its photocatalytic activity. The BiOI-G

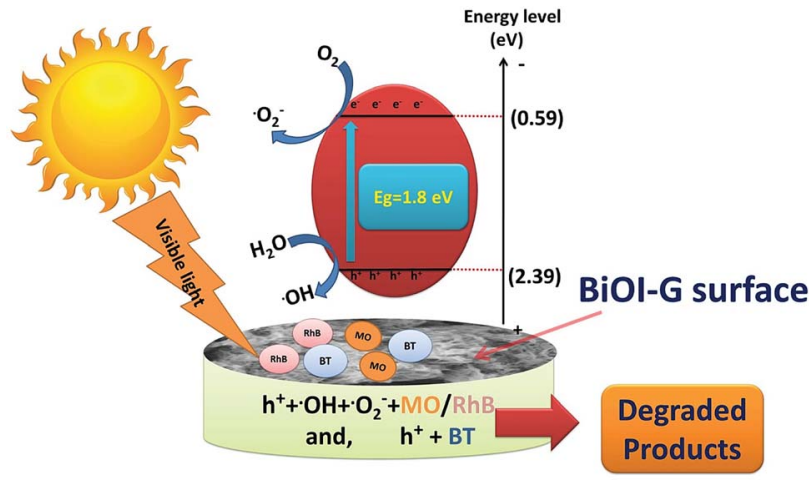

Fig. 7 Photocatalytic degradation mechanism for MO, RhB and BT.

displayed a narrower band gap than the BiOI-C sample, which favorably enhanced the electronic transition and boosted the formation of numerous electron-hole pairs. Based on the above analysis, all the factors are collectively playing a major role in the superior photocatalytic activity of BiOI-G.

On the basis of scavenger study and above discussion, a plausible mechanism for the degradation of $\mathrm{MO}, \mathrm{RhB}$, and $\mathrm{BT}$ was proposed. ${ }^{\mathbf{4 7}, 48}$ As shown in Fig. 7, under dark experiment, the organic pollutants get adsorbed on the surface of the photocatalyst. Upon visible light irradiation, BiOI-G is excited and electronic transition occurs at a rapid rate. The excited electrons in the $\mathrm{CB}$ react with the $\mathrm{O}_{2}$ molecules, which are adsorbed on the surface of BiOI-G to produce ${ }^{\cdot} \mathrm{O}_{2}{ }^{-}$, leave behind photogenerated $\mathrm{h}^{+}$in the valence band. The holes remaining in the VB react with the $\mathrm{H}_{2} \mathrm{O}$ molecules to produce $\mathrm{OH}$ radicals. The major active species i.e. ${ }^{\circ} \mathrm{OH},{ }^{\circ} \mathrm{O}_{2}{ }^{-}$and $\mathrm{h}^{+}$actively participates in the chemical reaction with the MO and $\mathrm{RhB}$ molecules to yield the degraded products. The photogenerated $\mathrm{h}^{+}$were the major active species that solely took part in the disintegration of BT.

\section{Conclusions}

In summary, a simple, ecofriendly and economically viable method was introduced to synthesize BiOI-G utilizing Azadirachta indica leaf extract. The phytochemicals present in the leaf extract controlled the size and provided stability to the as-prepared BiOIG. The absorption edge of BiOI-G shifted substantially towards the longer wavelengths compared to BiOI-C. BiOI-G possessed substantially larger surface area and porosity, which accounted for maximum photocatalytic active sites for the pollutants. Furthermore, the PL spectra, photocurrent response and EIS studies revealed the higher electronic transition and effectual separation of the photogenerated $\mathrm{e}^{-}-\mathrm{h}^{+}$pairs in BiOI-G. BiOI-G displayed superior photocatalytic performance for MO, RhB and BT degradation under visible source of light. This methodology provides a new direction towards an economical and ecofriendly route as an alternative for all chemical routes, which are detrimental to the environment safety.

\section{Conflicts of interest}

There are no conflicts to declare. 


\section{Acknowledgements}

This work is financially supported by DST, Government of India (INT/HUN/P-06/2016), NRDIO, Hungary (TÉT_15-IN-1-20160013) and WOG Technology, New Delhi, India. The authors acknowledge Enikő Bárdos, University of Szeged for assistance in HRTEM studies.

\section{Notes and references}

1 D. K. Tiwari, J. Behari and P. Sen, World Appl. Sci. J., 2008, 3, 417-433.

2 S. Jain and M. S. Mehata, Sci. Rep., 2017, 7, 15867.

3 P. Nagajyothi, T. M. An, T. Sreekanth, J.-i. Lee, D. J. Lee and K. Lee, Mater. Lett., 2013, 108, 160-163.

4 B. K. Bindhani and A. K. Panigrahi, J. Nanomed. Nanotechnol., 2015, S6, 008.

5 P. Banerjee, M. Satapathy, A. Mukhopahayay and P. Das, Bioresour. Bioprocess., 2014, 1, 3.

6 K. Saware and A. Venkataraman, J. Cluster Sci., 2014, 25, 1157-1171.

7 S. Yedurkar, C. Maurya and P. Mahanwar, Open J. Synth. Theory Appl., 2016, 5, 1-14.

8 A. Verma and M. S. Mehata, J. Radiat. Res. Appl. Sci., 2016, 9, 109-115.

9 S. Garg, M. Yadav, A. Chandra, S. Sapra, S. Gahlawat, P. Ingole, M. Todea, E. Bardos, Z. Pap and K. Hernadi, Materials, 2018, 11, 1273.

10 N. Jones, B. Ray, K. T. Ranjit and A. C. Manna, FEMS Microbiol. Lett., 2008, 279, 71-76.

11 G. Sangeetha, S. Rajeshwari and R. Venckatesh, Mater. Res. Bull., 2011, 46, 2560-2566.

12 T. Prathna, L. Mathew, N. Chandrasekaran, A. M. Raichur and A. Mukherjee, in Biomimetics learning from nature, InTech, 2010.

$13 \mathrm{~S} . \mathrm{Li}, \mathrm{S} . \mathrm{Hu}, \mathrm{K} . \mathrm{Xu}, \mathrm{W} . \mathrm{Jiang}, \mathrm{J} . \mathrm{Liu}$ and Z. Wang, Nanomaterials, 2017, 7, 22.

14 Y. Xiang, P. Ju, Y. Wang, Y. Sun, D. Zhang and J. Yu, Chem. Eng. J., 2016, 288, 264-275.

15 L. Chen, R. Huang, M. Xiong, Q. Yuan, J. He, J. Jia, M.-Y. Yao, S.-L. Luo, C.-T. Au and S.-F. Yin, Inorg. Chem., 2013, 52, 11118-11125.

16 Z. Jiang, X. Liang, Y. Liu, T. Jing, Z. Wang, X. Zhang, X. Qin, Y. Dai and B. Huang, Appl. Catal., B, 2017, 211, 252-257.

17 G. Dai, J. Yu and G. Liu, J. Phys. Chem. C, 2011, 115, 73397346.

18 Q. Teng, X. Zhou, B. Jin, J. Luo, X. Xu, H. Guan, W. Wang and F. Yang, RSC Adv., 2016, 6, 36881-36887.

19 H. Huang, Y. He, X. Du, P. K. Chu and Y. Zhang, ACS Sustainable Chem. Eng., 2015, 3, 3262-3273.

20 Y. Liu, W. Yao, D. Liu, R. Zong, M. Zhang, X. Ma and Y. Zhu, Appl. Catal., B, 2015, 163, 547-553.

21 Y. Feng, C. Liu, H. Che, J. Chen, K. Huang, C. Huang and W. Shi, CrystEngComm, 2016, 18, 1790-1799.

22 J. Luo, X. Zhou, L. Ma and X. Xu, J. Mol. Catal. A: Chem., 2015, 410, 168-176.
23 J. Cao, B. Xu, H. Lin, B. Luo and S. Chen, Dalton Trans., 2012, 41, 11482-11490.

24 J. Di, J. Xia, M. Ji, B. Wang, S. Yin, H. Xu, Z. Chen and H. Li, Langmuir, 2016, 32, 2075-2084.

25 R. Dong, Y. Hu, Y. Wu, W. Gao, B. Ren, Q. Wang and Y. Cai, J. Am. Chem. Soc., 2017, 139, 1722-1725.

26 L. Liu, Z. Liu, H. Bai and D. D. Sun, Water Res., 2012, 46, 1101-1112.

27 D. Hou, W. Luo, Y. Huang, C. Y. Jimmy and X. Hu, Nanoscale, 2013, 5, 2028-2035.

28 F. Duo, Y. Wang, C. Fan, X. Zhang and Y. Wang, J. Alloys Compd., 2016, 685, 34-41.

29 I. Sharma, G. K. Tripathi, V. K. Sharma, S. N. Tripathi, R. Kurchania, C. Kant, A. K. Sharma and K. Saini, Cogent Chem., 2015, 1, 1076371.

30 Y. Huo, Z. Xie, X. Wang, H. Li, M. Hoang and R. A. Caruso, Dyes Pigm., 2013, 98, 106-112.

31 X. Xiao and W.-D. Zhang, J. Mater. Chem., 2010, 20, 58665870.

32 M. Stan, A. Popa, D. Toloman, A. Dehelean, I. Lung and G. Katona, Mater. Sci. Semicond. Process., 2015, 39, 23-29.

33 J. Di, J. Xia, Y. Ge, L. Xu, H. Xu, J. Chen, M. He and H. Li, Dalton Trans., 2014, 43, 15429-15438.

34 W.-T. Sun, Y. Yu, H.-Y. Pan, X.-F. Gao, Q. Chen and L.-M. Peng, J. Am. Chem. Soc., 2008, 130, 1124-1125.

35 Y. Wang, K. Deng and L. Zhang, J. Phys. Chem. C, 2011, 115, 14300-14308.

36 J. Di, J. Xia, M. Ji, L. Xu, S. Yin, Q. Zhang, Z. Chen and H. Li, Carbon, 2016, 98, 613-623.

37 G. I. Waterhouse, G. A. Bowmaker and J. B. Metson, Phys. Chem. Chem. Phys., 2001, 3, 3838-3845.

$38 \mathrm{H}$. Yu, R. Liu, X. Wang, P. Wang and J. Yu, Appl. Catal., B, 2012, 111, 326-333.

39 J. Di, J. Xia, Y. Ge, H. Li, H. Ji, H. Xu, Q. Zhang, H. Li and M. Li, Appl. Catal., B, 2015, 168, 51-61.

$40 \mathrm{X}$. Gao, H. B. Wu, L. Zheng, Y. Zhong, Y. Hu and X. W. D. Lou, Angew. Chem., 2014, 126, 6027-6031.

41 Y. Xu and M. A. Schoonen, Am. Mineral., 2000, 85, 543-556. 42 H. Huang, Y. He, R. He, X. Jiang, Z. Lin, Y. Zhang and S. Wang, Inorg. Chem. Commun., 2014, 40, 215-219.

43 L. Zhang, H. B. Wu, Y. Yan, X. Wang and X. W. D. Lou, Energy Environ. Sci., 2014, 7, 3302-3306.

44 G. Liu, P. Niu, C. Sun, S. C. Smith, Z. Chen, G. Q. Lu and H.-M. Cheng, J. Am. Chem. Soc., 2010, 132, 11642-11648.

45 M. Guan, C. Xiao, J. Zhang, S. Fan, R. An, Q. Cheng, J. Xie, M. Zhou, B. Ye and Y. Xie, J. Am. Chem. Soc., 2013, 135, 10411-10417.

46 S.-W. Cao, X.-F. Liu, Y.-P. Yuan, Z.-Y. Zhang, Y.-S. Liao, J. Fang, S. C. J. Loo, T. C. Sum and C. Xue, Appl. Catal., B, 2014, 147, 940-946.

47 L. Ye, L. Zan, L. Tian, T. Peng and J. Zhang, Chem. Commun., 2011, 47, 6951-6953.

48 X. Zhang, C.-Y. Wang, L.-W. Wang, G.-X. Huang, W.-K. Wang and H.-Q. Yu, Sci. Rep., 2016, 6, 22800. 